\title{
A RFID Spatio-temporal Data Model Oriented Supply Chain
}

\author{
Wang Yonghui* and Qin Xiaoyu
}

School of Information \& Control Engineering, Shenyang Jianzhu University, Shenyang, 110168, China

\begin{abstract}
The supply chain which applies the RFID technology in real-time locating and tracking items not only results in saving plenty of time and space due to the RFID data, but also includes the implicit semantics. To implement efficient spatio-temporal queries, as well as locate and track the moving objects, this study develops a RFID supply chain-oriented spatio-temporal data model which defines the RFID data operations of spatio-temporal data. The space and time data manipulation methods are implemented in a relational database. Finally, these methods are operated using the experimental spatial and temporal data on RFID, with which the operating time can be analyzed to verify the validity of the data model and data manipulation.
\end{abstract}

Keywords: Database queries, RFID, Supply chain, Spatio-temporal data mode.

\section{INTRODUCTION}

RFID (Radio Frequency Identification) utilizes the wireless RF communication way to automatically achieve the non-contact type recognition technology, and can realize the real-time identifying, locating and tracking implementation in the supply chain. It has been widely used in the market. For example, Wal-Mart, as the global supermarket, has been requiring its main dealer to use the RFID label at the beginning of 2005, and Wal-Mart's decision has caused great concerns for RFID technology in logistics and supply chain.

The RFID technology obtains the identified item information from the readers by scanning the EPC tags to produce a large number of data with a tuple of the form (EPC, LOC, TIME). Herein, EPC is an Electronic Product Code which uniquely identifies the items or the packing containers; LOC is the location of the reader; TIME is the record time when the reader reads the item. According to the characteristics of RFID data, the management of RFID data is data cleaning [1,2], filtering [3,4] and compression [5]. The temporal data model [6] mainly uses the timestamp property to express the time of the event.

The data unit form shows that RFID data not only have the temporal characteristics, but also have the space character. RFID data can change as time and space change constantly. For instance, in the collection of the observations, the information with status changes, the items between locations and the items containing relationships will change over time. At the same time, in the practical application, readers and tags can be placed inside the personal digital assistants (PDAs), mobile phone or other mobile objects. The labeled items and reader are all moving. The RFID data are the spatio-temporal data. Therefore, after

*Address correspondence to this author at the No. 9, Hunnan East Road, Hunnan New District, Shenyang, China. Postcard: 110168; Tel: 86-13604039856; E-mail: yonghuiwang@sjzu.edu.cn the spatial and temporal characteristics of RFID data are fully considered, setting up a RFID spatio-temporal data model is of vital significance for the storage and management of RFID data.

This paper proposes a RFID data storage model based on the sequence of time and space. The model fully considers the characteristics of the supply chain oriented RFID data, discusses the relationships of the items between time and space in detail, adds the items' relationship on the geographical location, defines the query operation on the database, enables the model to query the basic information of the items, moves the path and related supply chain information, as well as effectively solves the tracking problem of the goods' information in the supply chain.

\section{RELATED WORK}

With the extensive use of RFID technology, there have been increasing researches on the RFID data, playing an important role in the supply chain application. However, according to the research of Feng Liang [7], the development prospects of RFID technology can help solve the problems in the supply chain, and meanwhile optimize the structure of the supply chain. Thus, RFID is of great significance in the supply chain.

The current research on RFID data can be divided into two aspects: one is the processing of RFID data [8-10]; second is how to establish the RFID data model. As the RFID data processing research has become more mature now, and the literature [1] presents a comprehensive data cleansing strategy based on the RFID application, all types of dirty data can be modified effectively; literature [3] proposes a type of RFID data stream filtering algorithm to filter the noise data and redundant data effectively; for RFID data compression, J. Han [5] puts forward two novel RFID data storage models, including the path cube and workflow cube to effectively and flexibly improve the analysis in the multidimensional space. 


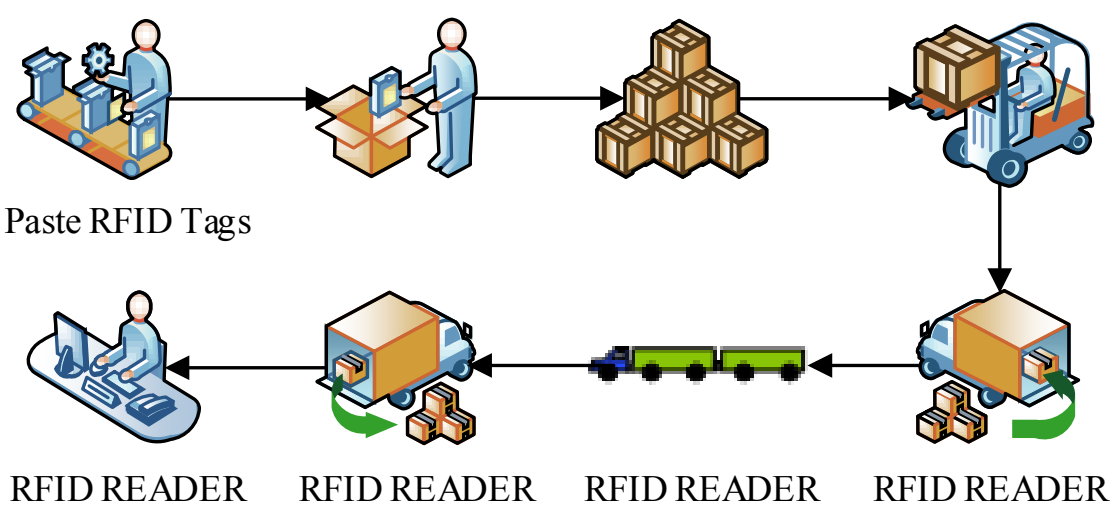

Fig. (1). A simple supply chain with RFID.

Due to the spatial and temporal characteristics of the RFID data, it is difficult to establish a data storage model. Lim puts forward a spatio-temporal data indexing scheme based on the trajectory of the query RFID [11]. Fan Yushun of Tsinghua University puts forward a complex event processing in the intelligent objects. RFID data are analyzed from the perspective of event handling relationship between space and time [12]. Li puts forward a RFID data processing method to analyze the relationship between time and space combined with the GIS and GPS technologies oriented in information fusion to solve the problem of RFID's inaccurate positioning [13]. These studies solve the dynamic relationship between time and location. However, there is no uniform definition for time and space. To a certain extent, the RFID data are ambiguous and inconsistent without unique features of time and space, comprehensively considering the temporal and spatial information model and the consistency control.

Hector Gonzalez, etc. [14] put forward a new gateway graph model which can be used to store a large number of RFID data related to transportation. The model has a very good effect on the large amounts of data produced by the moving objects.

Shaorong Liu [6] has carried out the detailed research referring to the literature [15] and the time series of the different types of RFID reader and tag. Through the analysis of the types of reader, a RFID temporal data model of supply chain is established. However, the position containment relationship and items containment relationship of this model are set in advance. It is a very big workload for the supply chain which has massive moving objects.

\section{A RFID SPATIO-TEMPORAL DATA MODEL ORIENTED SUPPLY CHAIN}

\subsection{RFID Spatio-temporal Data Model}

In the supply chain, RFID technology is used to locate and track the object. Such process produces a lot of spatiotemporal sequence like the form (EPC, LOC, TIME). This

paper based on the location information of items carries out the detailed research, supplements the location relation- ship, and analyzes the relationship between the adjacent relation and the superior location. The path information of the goods is also added to the model to establish a supply chain oriented in the RFID spatio-temporal data model.

Fig. (1). shows a simple RFID application of the supply chain. The items are packed into one container. The containers are put on the trucks, and transported to the destination to finally reach the consumers. In each process, there is a reader to read the label information.

According to the items in the supply chain moving mode, reader reads the information, the uniqueness of tag ID, and the characteristics of the data generated in the RFID in the supply chain. The spatial-temporal data model is shown in Fig. (2).

The above model is the entity data model. The OBSERVATION table stores the original information of the items, including reader's epc (Electronic Product Code), tag's epc and the reading timestamp; the OBJECT table records the basic information on object's epc, name, type, manufacturer and the price of the epc-tagged object; the READER table stores the reader epc, name, location id (which presents the position about the reader) and the description of the reader. The reader_epc is the primary key, and location_id is the foreign key associated with the entity table LOCATION. A sample example of table LOCATION is shown in Table $\mathbf{1}$.

The LOCATION table records the symbolic location in track, and the PATH table records the path information of moving object, including the path_id and the direction of the path. The PATH table, as the supplement on the location, illustrates the relationship between locations which is essential to information query. A sample of table PATH is shown in Table 2.

The direction distribution in Table 2 represents the moving direction of the path from the root node to the leaf node. The PATH table plays a vital role in finding the primary path of the transportation and the sequence path to obtain the directions of the moving items. The sequence path can be used by the company to make decision on the product research and development, product distribution and product transportation. It is very helpful for the enterprise management. 


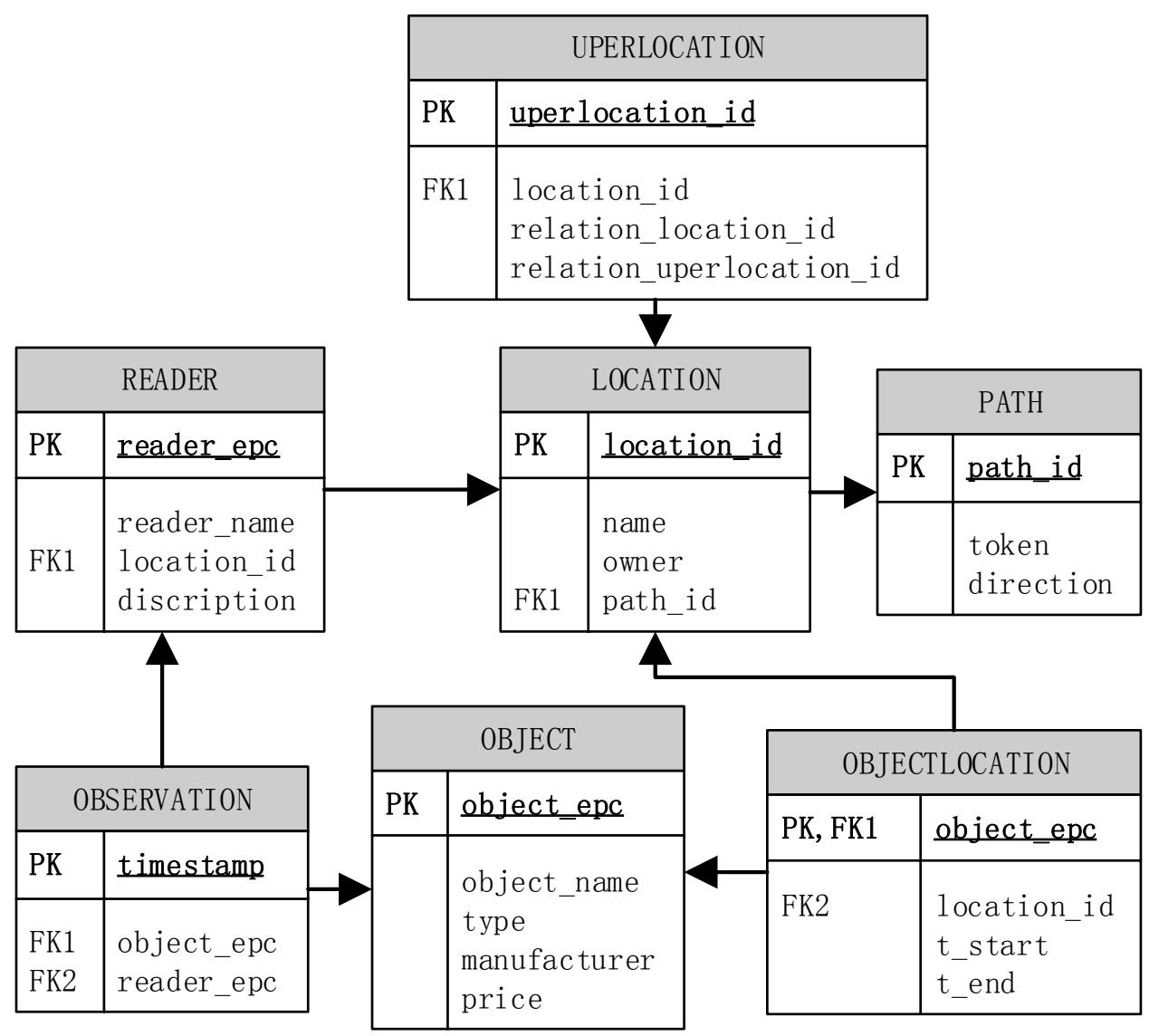

Fig. (2). RFID spatio-temporal data model.

Table 1. Location table.

\begin{tabular}{|c|c|c|}
\hline Location_id & Name & Owner \\
\hline \hline LOCA & Production Line E & Manufacturer D \\
\hline LOCB & Warehouse A & Carrier B \\
\hline LOCC & Route to Retail C & Retailer C \\
\hline LOCD & Retailer Store C & Customer \\
\hline LOCE & Customer & \\
\hline
\end{tabular}

Table 2. Path table.

\begin{tabular}{|c|c|c|}
\hline Path_id & Token & Direction \\
\hline \hline 1 & 1,2 & True \\
\hline 2 & 2,3 & True \\
\hline 3 & 1,3 & True \\
\hline 4 & $1,2,3$ & True \\
\hline 5 & $1,2,3,4$ & True \\
\hline 6 & 1.3 .4 & True \\
\hline
\end{tabular}


Table 3. Upper level location table.

\begin{tabular}{|c|c|c|c|}
\hline $\begin{array}{c}\text { Upper } \\
\text { Location_id }\end{array}$ & Location_id & $\begin{array}{c}\text { Relation_ } \\
\text { Location_id }\end{array}$ & $\begin{array}{c}\text { Relation_ } \\
\text { Upper Level Location_id }\end{array}$ \\
\hline \hline ULOCA & LOCA & LOCB & ULOCC \\
\hline ULOCA & LOCE & LOCF LOCA \\
\hline ULOCB & LOCB & LOCD & ULOCD \\
\hline ULOCC & LOCC & LOCC & ULOCA ULOCB \\
\hline ULOCD & LOCD & LOCE & ULOCB \\
\hline ULOCD & LOCF & & LLCB \\
\hline
\end{tabular}

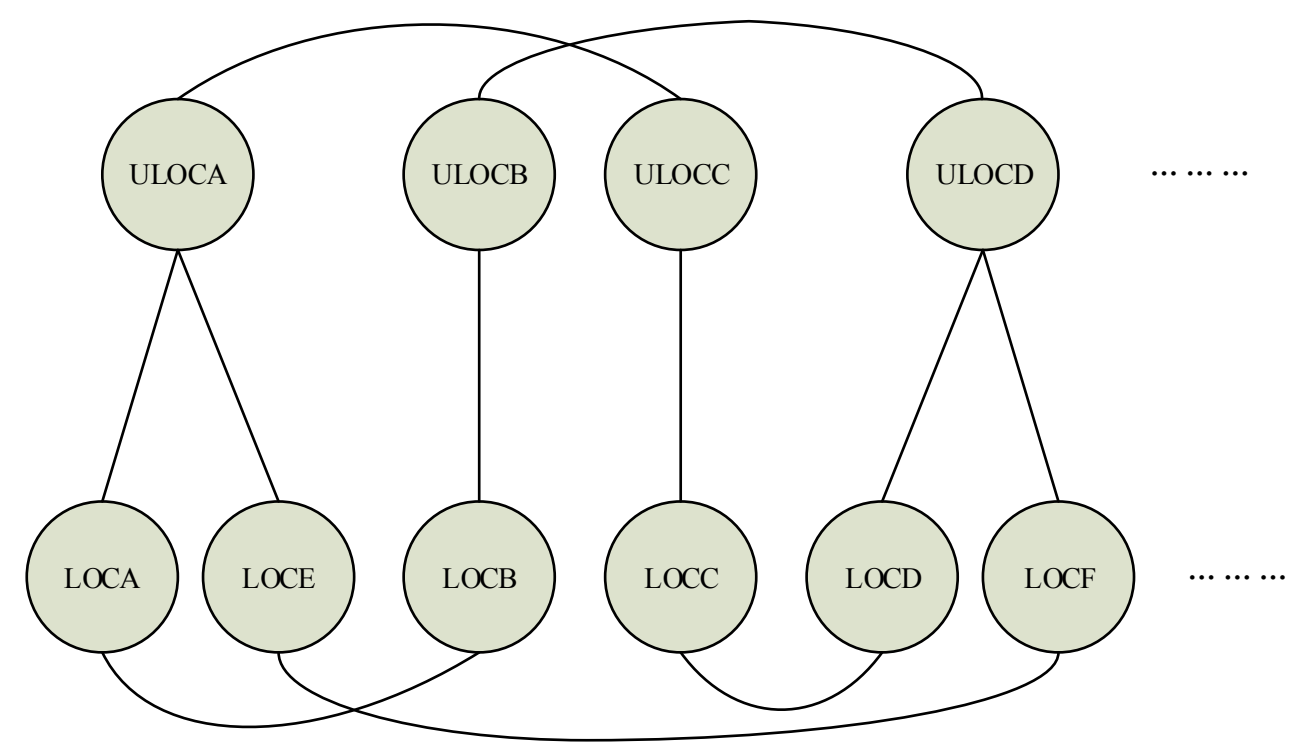

Fig. (3). An instruction of the table upper level location

Another supplement on the location information is to add a related location information and an upper level location information that can be associated with the symbolic location, and use the upper level location table to store the information. This can make it easier to find the next level item information. The upper level location table includes the location id, relation location id, upper level location id and relation upper level location id. A sample of table UPPER LEVEL LOCATION is presented in Table 3.

This table is divided into two parts: the first one is to put the query on the location information; the other one is the upper level location id which represents the higher level of the distribution station. In the supply chain, there are a lot of distribution stations which can be divided into different levels of distribution centers to show the particular status. Thus, it plays an important role in the later study on the location data mining. A simple diagram is shown in Fig. (3): the high-level location and the low-level location have the relation locations.

The objectlocation table records the item's epc, location_id and the time while the items stay in one location, start time and end time. A sample of the table OBJECTLOCATION is presented in Table 4.
Another important relationship table in the database is CONTAINMENT table stored in the item's epc and during the time [t_start, t_end], which includes the item's epc, namely object_parent_epc. A sample of the table CONTAINMENT is presented in Table $\mathbf{5}$.

The table represents the containment relationship usually set in advance, and can quickly find the source and the items with the tested items at the same time and at the same place. If the production has some problem, it can quickly find its producers, transportation vehicles and other productions produced in the same factory or transported in the same truck.

\subsection{RFID Spatio-temporal Data Query Operation}

In the supply chain, RFID data's query in the database on the final can be divided into three categories:

(1) The electronic product code query, namely query item's epc, is mainly divided into simple query in a single table and complex queries in more than one table;

(2) The item's location information query, namely query item's location_id, needs the join operations between the tables; 
Table 4. Objectlocation table.

\begin{tabular}{|c|c|c|c|}
\hline Object_epc & Location_id & t_start & t_end \\
\hline \hline 1.2 .2 & LOCA & 2013.05 .01 & 2013.07 .01 \\
\hline 1.2 .3 & LOCA & 2013.05 .07 & 2013.06 .30 \\
\hline 1.2 .1 & LOCA & 2013.07 .20 & UC \\
\hline 1.2 .2 & LOCB & 2013.07 .02 & UC \\
\hline 1.2 .3 & LOCB & 2013.07 .01 & 2013.12 .14 \\
\hline 1.2 .4 & LOCB & 2013.10 .25 & UC \\
\hline 1.2 .1 & LOCC & 2013.09 .20 & UC \\
\hline 1.2 .5 & LOCC & 2014.01 .26 & UC \\
\hline 1.2 .4 & LOCC & 2013.12 .25 & U \\
\hline
\end{tabular}

Table 5. Containment table.

\begin{tabular}{|c|c|c|c|}
\hline Object_epc & $\begin{array}{c}\text { Parent_ } \\
\text { Object_epc }\end{array}$ & t_start & t_end \\
\hline \hline 1.2 .1 & 10.1 .2 & $2013: 10: 20$ & $2013: 10: 24$ \\
\hline 1.2 .2 & 10.7 .3 & $2013: 09: 10$ & $2013: 09: 20$ \\
\hline 1.2 .3 & 11.3 .1 & $2013: 11: 01$ & $2013: 12: 10$ \\
\hline 1.2 .4 & 12.5 .4 & $2013: 12: 03$ & $2013: 12: 30$ \\
\hline 1.2 .5 & 14.2 .5 & $2013: 12: 25$ & $2014: 01: 12$ \\
\hline 1.2 .6 & 13.2 .6 & $2014: 01: 02$ & \\
\hline
\end{tabular}

(3) The item's path information query, namely query item path_id, also needs the join operations between the tables.

According to the above queries, the table related to this application is: the OBJECT table, the LOCATION table, PATH table, UPPER LEVEL LOCATION table and OBJECTLOCATION table. The following definition uses the first letter instead of the above four kinds of tables. Through the OBL representative OBJECTLOCATION table, the data model is shown in the Fig. (2) on the database query.

\subsubsection{Definition A (Selection)}

The selection operation on the relation $\mathrm{R}$ is defined as $\operatorname{Sel}_{c}(R)=\left(R, S^{\prime}\right)$. In $S^{\prime}=\{L, O B L \infty O\}$, when $S^{\prime}=L$, it is the simple query in the first category; when $S^{\prime}=O B L \infty O$, it is the complex query in the first category. In this definition, we illustrate the condition that $S^{\prime}=L$. If we select the information about the items where location id = 'LOCA', we can select the operation on the LOCATION table, and the expression is $\sigma_{\text {location } i d={ }^{L O C A}}(L)$.

\subsubsection{Definition B (Projection)}

The projection operation on the relation $\mathrm{R}$ is defined as $\operatorname{Proj}_{S^{\prime}}(R)=\left(R, S^{\prime}\right)$. In $S^{\prime}=\{L, U, P, L \infty U, L \infty P\}$, when $S^{\prime}=\{L, U, L \infty U\}$, the query is for the location information; when $S^{\prime}=\{L, P, L \infty P\}$, the query is for the path information. If the operation is conducted as follows: selects location_id from upper level location where upper level location id = 'ULOCA', it is necessary to do the projection operation on the upper level location table, and the expression is $\pi_{\text {location id }}\left(\sigma_{\text {uverlocation id }=, U L O C A}(U)\right)$.

\subsubsection{Definition C(Join)}

The join operation on the relation $R$ is defined $R \infty S=\left(R^{\prime}, S\right)$ In $R^{\prime}=\{O B L, L, P\}, \quad S^{\prime}=\{O, L \infty U, L \infty P\}$, when $S^{\prime}=O$, $R \propto S=O B L \infty O$ (there is no relationship among $\mathrm{L}, \mathrm{P}, \mathrm{O}$ ), it is the join operation between the two tables; when $S^{\prime}=\{L \infty U, L \infty P\}$, the join operation between $\mathrm{R}$ and $\mathrm{S}$ can be divided into two conditions: the first one is when $R^{\prime}=O B L, R \propto S=O B L \infty\{L \infty U, L \infty P\}$, it is the join operation among the three tables; the second one is when $R^{\prime}=\{L, P\}, R \infty S=\operatorname{Proj}_{C}(\{L, P\},\{L \infty U, L \infty P\})$, where C is the projection condition. Herein, the join operation be- 
Table 6. The result table.

\begin{tabular}{|c|c|c|c|c|}
\hline Object_epc & Location_id & $\begin{array}{c}\text { Relation_ } \\
\text { Location_id }\end{array}$ & t_start & t_end \\
\hline 1.2 .2 & LOCA & LOCB & 2013.05 .01 & 2013.07 .01 \\
\hline 1.2 .3 & LOCA & LOCB & 2013.05 .07 & 2013.06 .30 \\
\hline 1.2 .1 & LOCA & LOCB & 2013.07 .20 & 2013.09 .15 \\
\hline 1.2 .2 & LOCB & LOCA & 2013.07 .02 & $\mathrm{UC}$ \\
\hline 1.2 .3 & LOCB & LOCA & 2013.07 .01 & $\mathrm{UC}$ \\
\hline 1.2 .4 & LOCB & LOCA & 2013.10 .25 & 2013.12 .14 \\
\hline 1.2 .1 & LOCC & LOCD & 2013.09 .20 & UC \\
\hline 1.2 .5 & LOCC & LOCD & 2014.01 .26 & $\mathrm{UC}$ \\
\hline 1.2 .4 & LOCC & LOCD & 2013.12 .25 & UC \\
\hline
\end{tabular}

tween $\mathrm{R}$ and $\mathrm{S}$ becomes the join operation between the two tables first and then conduct the projection operation among them by condition $\mathrm{C}$.

Herein, the first condition is operated as an example. Suppose, there is such a query: object epc is selected from object location, location, and upper level location where the relation_location_id = 'LOCB' AND t_end = 'UC'. The LOCATION $\bar{N}$ table and UPPER LEVEL LOCATION table are first joined to obtain the table, including the attribution (location_id and relation_location_id). Subsequently, the new table along with the OBJECTLOCATION table is provided. The operation result is shown in Table 6.

\subsection{Data Updating and Query Optimization}

For data update plays an essential role in updating the latest data, the data are updated according to the real-time properties. It can be divided into two categories. One kind is t_end != ' $\mathrm{UC}$ ', namely the data at the end of the time. Thus, it is important to obtain the latest update of the data. Another type is t_end = ' $U C$ '; yet ( $\mathrm{t}$-end - $\mathrm{t}$ _start) is greater than a certain time interval. It shows that such type of items is staying for a long time in one place. Considering the inherent characteristics of items, it should be disposed in time.

The basic SQL language is used for data query, except the optimization of the items that include the relationship. A definition is also made on the basic type of the item query, so that it is more convenient to query all types of information about the items. In addition, the hierarchy is added to the location information, which is not only convenient for defective products, but also has important significance on the analysis of the goods flow and optimization of industrial structure in the supply chain.

\section{EXPERIMENTS}

The experimental environment of this experiment is based on the Windows 7 system. The memory is $2 \mathrm{~GB}$, and the link uses the Microsoft SQL Server 2008, based on the experimental data of the supply chain of synthetic data.

The experiment is conducted in three parts, including comparison of query time, the comparison of execution time and update times and the comparison of storage space. All the results are compared with the two methods, including the original method and the containment method.

The following queries are shown in Table 7. According to the model, to find out whether there exists the containing relationship, the average query time should be analyzed.

The detailed query process is as follows.

Q1. Find whether the object_epc='1.2.3' in the store.

\section{SELECT *}

\section{FROM OBJECTLOCATION}

WHERE object_epc $=$ '1.2.3'

AND location_id = 'LOCB'

AND t_end = 'UC'

Q2. Count the numbers of the object at location A during the time from $t$ _start to $t$ _end.

\section{SELECT COUNT(*)}

FROM OBJECTLOCATION 
Table 7. The queries.

\begin{tabular}{|c|c|c|}
\hline & The Queries & The Number of Queries \\
\hline \hline 1 & Q1- Whether an object in a certain position & 50 \\
\hline 2 & Q2-At one minute, the number of the objects in a position & 10 \\
\hline 3 & Q3-The objects with a particular object in the same position & 18 \\
\hline 4 & Q4-The source of the object & 22 \\
\hline 5 & Q5-The objects that are distributed in one position & 26 \\
\hline
\end{tabular}

Table 8. Containment C1.

\begin{tabular}{|c|c|c|c|}
\hline Object_epc & $\begin{array}{c}\text { Parent_ } \\
\text { Object_epc }\end{array}$ & t_start & t_end \\
\hline \hline 1.2 .1 & 10.1 .2 & $2013: 10: 20$ & $2013: 10: 24$ \\
\hline 1.2 .2 & 10.7 .3 & $2013: 09: 10$ & $2013: 09: 20$ \\
\hline 1.2 .3 & 11.3 .1 & $2013: 11: 01$ & $2013: 11: 09$ \\
\hline 1.2 .4 & 12.5 .4 & $2013: 12: 03$ & $2013: 12: 10$ \\
\hline 1.2 .5 & 14.2 .5 & $2013: 12: 25$ & $2013: 12: 30$ \\
\hline 1.2 .6 & 13.2 .6 & $2014: 01: 02$ & $2014: 01: 12$ \\
\hline 1.2 .7 & 15.2 .6 & $2013: 11: 22$ & $2013: 11: 29$ \\
\hline 1.2 .8 & 17.2 .3 & $2014: 01: 22$ & $2014: 01: 30$ \\
\hline
\end{tabular}

Table 9. Containment C2.

\begin{tabular}{|c|c|c|c|}
\hline Object_epc & $\begin{array}{c}\text { Parent_ } \\
\text { Object_epc }\end{array}$ & t_start & t_end \\
\hline \hline 1.3 .1 & 10.1 .2 & $2013: 10: 20$ & $2013: 10: 24$ \\
\hline 1.3 .2 & 11.7 .3 & $2013: 09: 15$ & $2013: 11: 19$ \\
\hline 1.3 .3 & 12.3 .1 & $2013: 11: 11$ & $2013: 12: 10$ \\
\hline 1.3 .4 & 12.5 .4 & $2013: 12: 03$ & $2013: 12: 30$ \\
\hline 1.3 .5 & 13.2 .5 & $2013: 12: 25$ & $2014: 01: 12$ \\
\hline 1.3 .6 & 14.2 .6 & $2014: 01: 02$ & $2014: 01: 07$ \\
\hline 1.3 .7 & 14.3 .5 & $2013: 11: 20$ & $2013: 11: 29$ \\
\hline
\end{tabular}

WHERE location_id = 'LOCA'

AND t_start = '2013-10-01 00:00:00'

AND t_end = '2013-12-31 00:00:00'

Q3. Find the other objects that being with the particular object. (The basic information is shown in Table 8 and Table 9)

SELECT c2.object_epc

FROM CONTAINMENT c1, CONTAINMENT c2
WHERE c1.object_parent_epc $=$ c2.object_parent_epc AND c1.object_epc $=$ '1.2.5' AND overlaps

(c1.tstart, c1.tend, c2.tstart, c2.tend)

Q4. Find the original of the objects.

SELECT 1.location_id, 1.object_epc

FROM OBJECTLOCATION1 


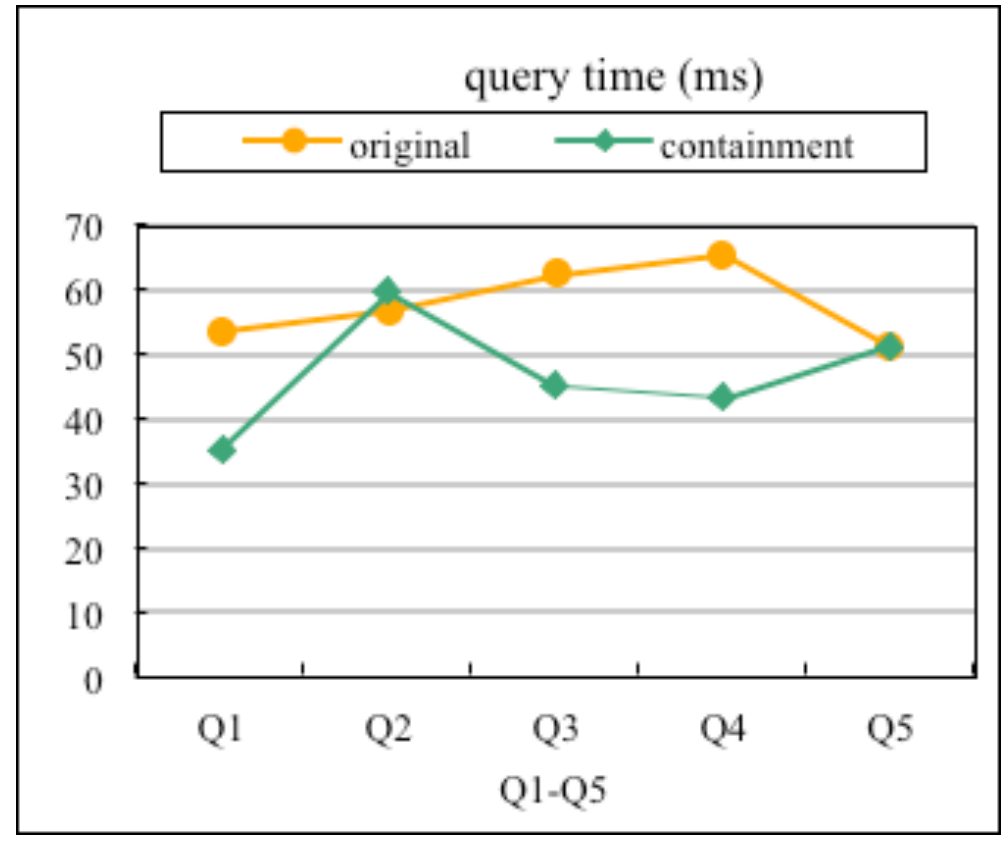

Fig. (4). The query result.

WHERE overlaps

(1.tstart, 1.tend, c2.tstart, c2.tend)

AND 1.object_epc in

(SELECT DISTINCT c2.object_epc

FROM CONTAINMENT c1, CONTAINMENT c2

WHERE c1.object_parent_epc $=$ c2.object_parent_epc

AND c1.object_epc $=$ '1.2.4'

AND overlaps

(c1.tstart, c1.end, c2.tstart, c2.tend))

Q5. Find the objects distributed from the location A and its relation location.

SELECT object_epc

FROM OBJECTLOCATION

WHERE location_id IN

(SELECT location_id

\section{FROM UPPER LEVEL LOCATION}

WHERE upper level location_id = 'ULOCA')

AND relation_location_id = 'LOCB')

The result of the query is shown in Fig. (4).

In Fig. (4), the original represents the ordinary query which does not have the containing relationship; and containment represents the query which has the containing relationship. It can be seen from Fig. (4): As Q1 queries with containing relationship, the number of data in a table are smaller than the data of the original method. Thus, the query time is smaller than the original method. In Q2 que- ry, the original method is to scan the table and sum it as the query results. However, the containment query needs to pack the containing object number through the outer packing and then sum it; thus, it is longer than the original query. Q3 and Q4 query have the containing relation; hence, the query time is shorter than the original query. Q5 query is based on the location query and the containment relationship does not involve the position; thereby, the two kinds of query time have no difference. Thus, for the supply chain with massive data, the containment relationship can reduce the query time and decrease the operations.

The experimental data of different population sizes are summarized. Herein, $\mathrm{m} 1$ is the biggest one, $\mathrm{m} 2$ is smaller than $\mathrm{m} 1$, and $\mathrm{m} 3$ is the smallest. Fig. (5) shows the result of the update times and Fig. (6) indicates the result of the execution time.

Fig. (5) shows that the update time of the original method is larger than the containment method. It shows that based on the condition that the data is moving, the containment method will be used to reduce the number of updates in the database.

Fig. (6) indicates that the execution time of the containment method is much smaller than the original method, the characteristic of the collective moving of the data is also adjusted according to the execution time.

But in the Fig. (5) and Fig. (6), a phenomenon of the data reduction in the number of collective moving, the update times and the execution of the original method are slipped down quickly, but the change of the method of the containment is not obvious. This shows that when the collective moving character of the RFID data is not strong, the use of the containment method to process the 


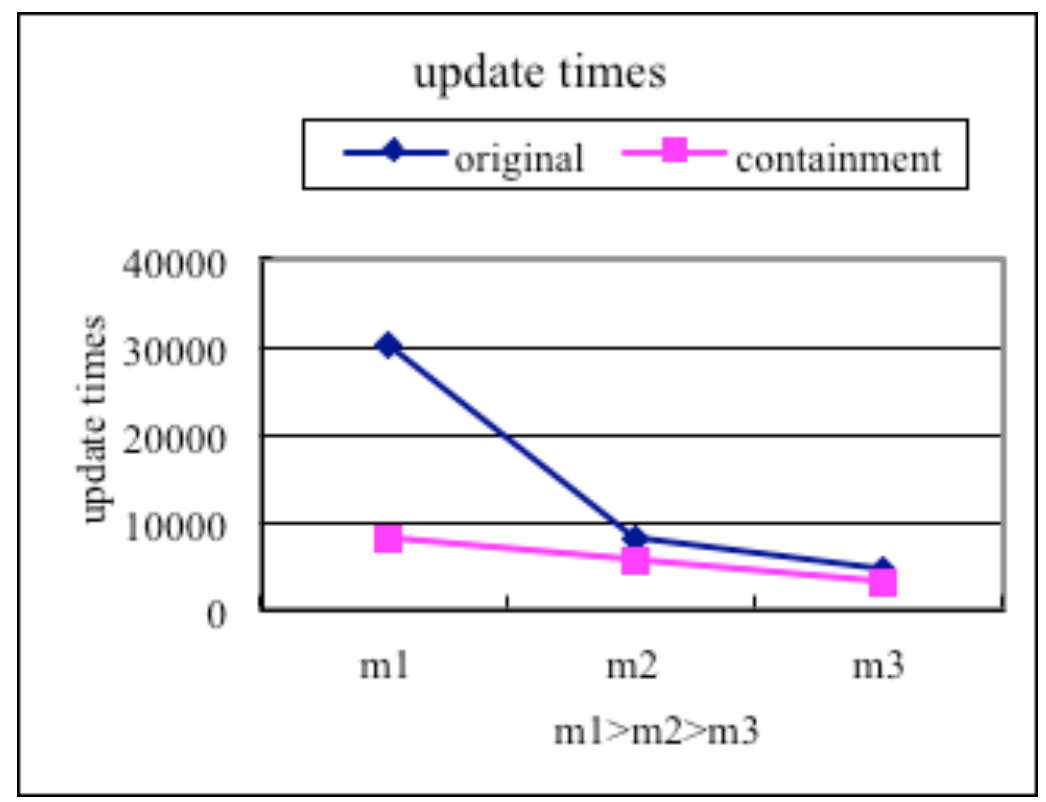

Fig. (5). The result of update times.

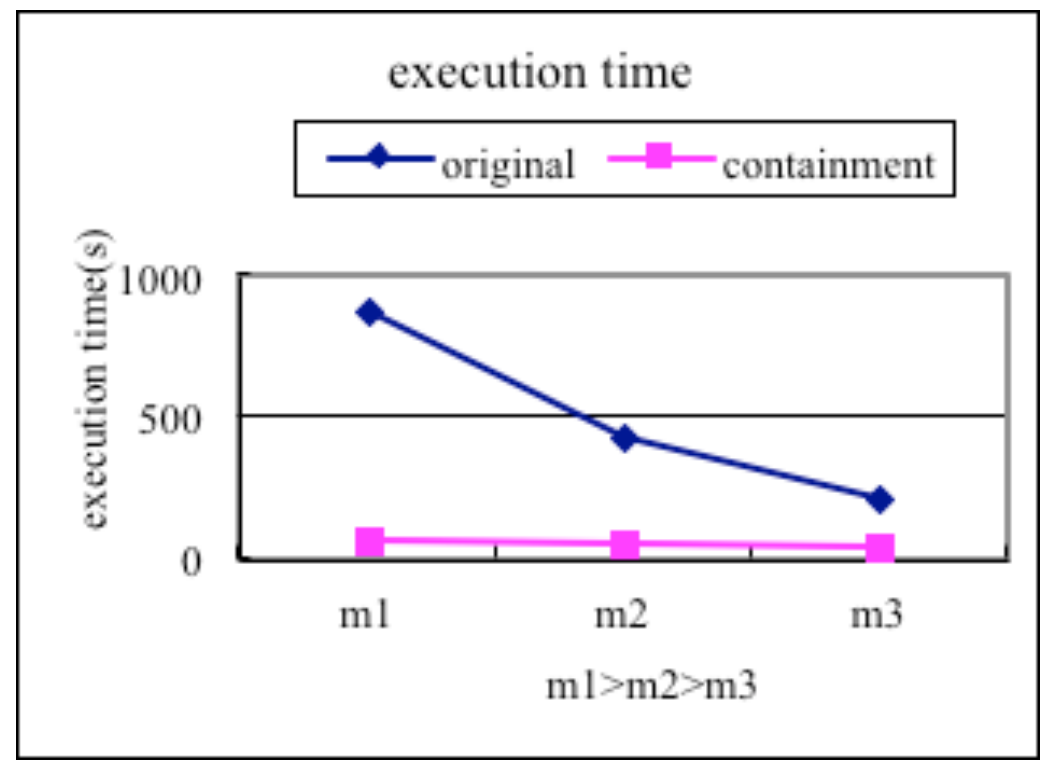

Fig. (6). The result of execution time.

data is not convenient. Thus, if the method of the containment can speed up the query time and reduce the update times, the characteristics of the data must be massive and moving.

Fig. (7) shows the comparison of the storage space.

In Fig. (7), the method of the original and the containment is on the count of $\mathrm{m} 1$. The conclusions are as follows:

Firstly, the containment method and the original method have no effect on the containment form as the two methods for the same data containing relationship are the same; for object location form, the collective motion features are used to reduce the record number, thus reducing the storage space.

Secondly, as the object motion is captured, the characteristics of the two forms of storage space are becoming more obvious. In comparison with the same amount of data, the outer packing contains fewer containment objects. As a result, the outer packing quantity and the number of location change are increasing. Thus, the two forms of storage space are becoming larger than before.

\section{CONCLUSION AND FUTURE WORK}

In the supply chain, the RFID technology can realize the positioning and tracking of the item. Based on the char- 


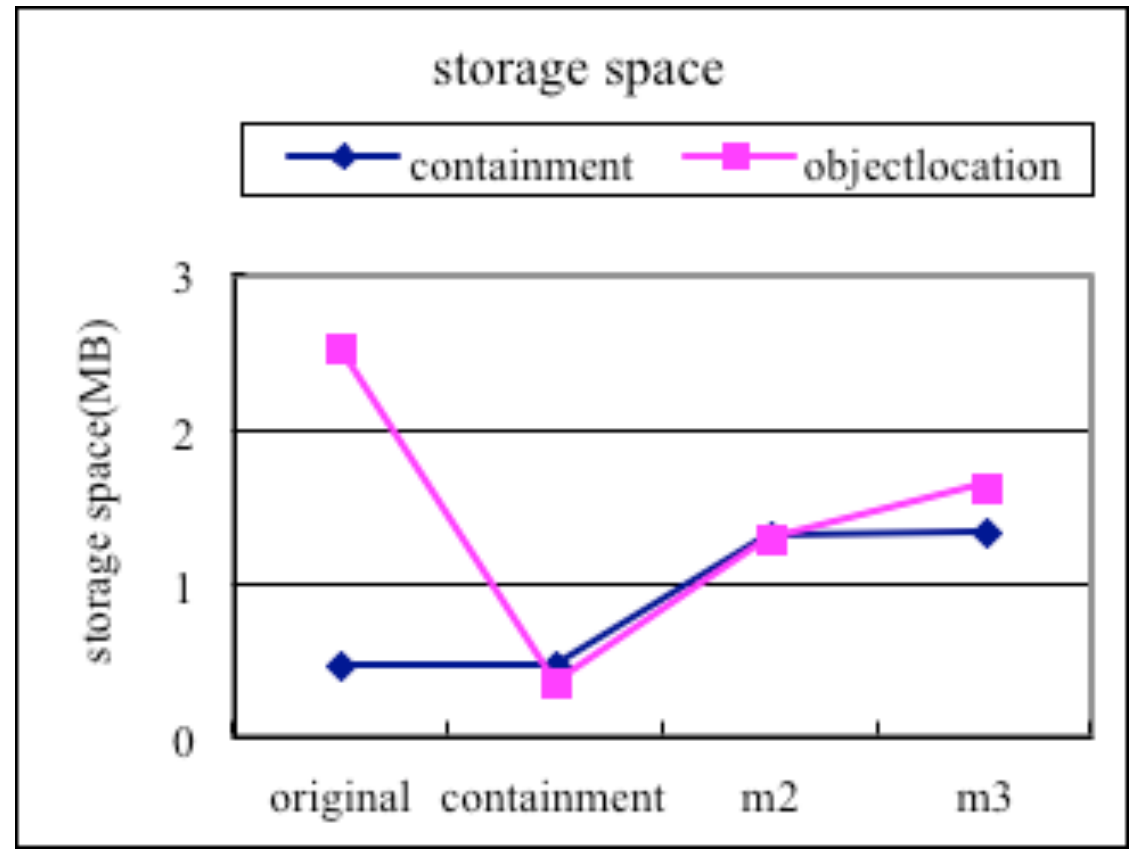

Fig. (7). The result of storage space.

acteristics of RFID data generated in the supply chain, this study sets up a RFID spatio-temporal data model oriented in supply chain, and adds the spatial location information to provide support for spatial information query and analysis. In the aspect of object information with basic information, this paper can determine the basic information of the query items to test whether the items are correct. However, in the path of multidimensional data mining, it also plays a vital role. On the basis of the established model, the data query is carried out, and the operation on the database is defined. The data query is very convenient.

In the experiment section, we have conducted three groups of experiments. The first experiment in the condition of object moving shows that the method of the containment is efficient; the second experiment shows the execution time and update time between the original method and the containment method. If there is a massive object in the containment relationship, the containment method is very effective for the query. The third experiment shows the storage of the two methods. The object is massive moving, showing that the containment can save the storage space for the database. Through the experiment, the method of the containment is efficient when the objects are moving together and the number of objects is massive.

The further study is based on the spatio-temporal data model which takes advantage of the location information and path information for data mining. This paper intends to use the graph to process the location information, and aims to analyze the path information from two aspects, including frequent path and multidimensional frequent path, per the number of occurrence of the path. In this study, the information of frequent path is used to analyze the commodity distribution problems in the supply chain and enterprises to contribute to the enterprise management and supply chain optimization.

\section{CONFLICT OF INTEREST}

The authors confirm that the content of this article has no conflict of interest.

\section{ACKNOWLEDGEMENTS}

First and foremost, I would like to show my deepest gratitude to the Education Department of Liaoning province "Science and Technology Research Projects (L2012212)" for the financial support to our project.

Secondly, I want to deliver my sincere thanks to my coauthor for the great efforts devoted to this work. I also want to show my gratitude to those who contributed to the research in this field and the scholars who have given me a lot of help and inspiration.

\section{REFERENCES}

[1] Y. Gu, X. Li, Y. Lü, and G. Yu, "Integrated Data Cleaning Strategy Based on RFID Applications", J. Northeast. Univ. Nat. Sci. (Natural Science), vol. 130, no. 1, pp. 35-37, Jan. 2009.

[2] X. Chen, X. Wang, and M. Ke, "An Improved Algorithm in Redundant Data Cleaning Based on RFID Middleware", MCC, vol. 30, no. 7, pp. 154-158, July, 2013.

[3] H. Deng, and J. Li, "Research of RFID data filtering algorithm", Comput. Technol. Develop., vol. 22, no. 6, pp. 26-29, June 2012.

[4] H. Jia, and W. Li, "Research on RFID Data Filtering Model for Warehouse Management", Comput. Appl. Softw., vol. 31, no. 2, pp. 74-76, Feb. 2014.

[5] J. Han, H. Gonzalez, X. Li, and D. Klabjan, "Warehousing and Mining Massive RFID Data Sets”, 2006, ADMA, LNAI 4093, pp. $1-18$.

[6] S. Liu, F. Wang, and P. Liu, "A Temporal RFID Data Model for Querying Physical Objects", A Timecenter Technical Report, pp.68, TR-88, February 8, 2007.

[7] L. Feng, Research on Supply Chain Warehouse Management and System Design based on RFID, Jinan: Shan Dong University, vol. 3, pp. 26-28, 2010. 
[8] O. Mylyy, "RFID Data Management, Aggregation and Filtering", Hasso Plattner Institute at the University of Potsdam, Prof.-Dr.Helmert-Strasse 2-3, Ed. 14482 Potsdam, Germany, 2006, pp. 6154.

[9] R. Derakhshan, M.E. Orlowska, and X. Li, "RFID Data Management: Challenges and Opportunities", In: IEEE Int. Conf. RFID Gaylord Texan Resort, Grapevine, TX, USA, 2007 March 26-28, pp. $175-182$.

[10] Y. Bai, F. Wang, P. Liu, C. Zaniolo, and S. Liu, RFID Data Processing with a Data Stream Query Language, Department of Computer Science UCLA, pp. 1184-1193, 2007.

[11] D. Lim, B. Hong, and D. Cho, "The Self-relocating Index Scheme for Telematics GIS", In: $5^{\text {th }}$ Int. workshop on W2GIS 2005, Lausanne, Switzerland, December 15-16, pp. 93-103, 2005.
[12] C. Zang, and Y. Fan, "Information system of manufacturing enterprise based on smart items", Comput. Integr. Manu. Syst., vol. 13, no. 1, pp. 49-56, 2007

[13] B. Li, and W. Li, "Research on RFID Oriented Information Fusion in Intelligent Logistics", J. Univ. Sci. Technol. C, vol. 36, no. 6, pp- 1329-1332, 2007.

[14] H. Gonzalez, J. Han, H. Cheng, X. Li, D. Klabjan, and T. Wu, "Modeling Massive RFID Data Sets", IEEE Trans. Knowl. Data Eng., vol. 22, no. 1, Jan, pp. 90-104, 2010

[15] F. Wang and P. Liu, "Temporal Management of RFID Data", VLDB, pp. 1128-1139, 2005.

Received: September 16, 2014

Revised: December 23, 2014

Accepted: December 31, 2014

(C) Yonghui and Xiaoyu; Licensee Bentham Open.

This is an open access article licensed under the terms of the Creative Commons Attribution Non-Commercial License (http://creativecommons.org/licenses/ by-nc/4.0/) which permits unrestricted, non-commercial use, distribution and reproduction in any medium, provided the work is properly cited. 